\title{
Psoriasis: a brief overview
}

\author{
Authors: Antony Raharja, ${ }^{\mathrm{A}}$ Satveer $\mathrm{K} \mathrm{Mahil}^{\mathrm{B}}$ and Jonathan N Barker ${ }^{\mathrm{C}}$
}

Psoriasis is a clinically heterogeneous lifelong skin disease that presents in multiple forms such as plaque, flexural, guttate, pustular or erythrodermic. An estimated 60 million people have psoriasis worldwide, with $1.52 \%$ of the general population affected in the UK. An immune-mediated inflammatory disease, psoriasis has a major genetic component. Its association with psoriatic arthritis and increased rates of cardiometabolic, hepatic and psychological comorbidity requires a holistic and multidisciplinary care approach. Psoriasis treatments include topical agents (vitamin D analogues and corticosteroids), phototherapy (narrowband ultraviolet $B$ radiation (NB-UVB) and psoralen and ultraviolet A radiation (PUVA)), standard systemic (methotrexate, ciclosporin and acitretin), biologic (tumour necrosis factor (TNF), interleukin (IL)-17 and IL-23 inhibitors) or small molecule inhibitor (dimethyl fumarate and apremilast) therapies. Advances in the understanding of its pathophysiology have led to development of highly effective and targeted treatments.

\section{Introduction}

Psoriasis is a lifelong immune-mediated inflammatory skin disease, associated with morbidities such as psoriatic arthropathy, psychological, cardiovascular and hepatic diseases. In 2014 , the World Health Organization recognised psoriasis as a serious non-communicable disease and highlighted the distress related to misdiagnosis, inadequate treatment and stigmatisation of this disease. The Global Burden of Disease Study estimated that psoriasis accounted for 5.6 million all-age disability-adjusted life-years (DALYs) in 2016; at least three-fold that of inflammatory bowel disease. ${ }^{2}$

\section{Epidemiology}

Psoriasis affects both males and females, with earlier onset in females and those with a family history. Its age of onset shows a bimodal distribution with peaks at 30-39 years and 60-69 years in men, and 10 years earlier in women. ${ }^{3}$

Authors: Afoundation year-2 doctor (academic foundation programme), St John's Institute of Dermatology, London, UK; ${ }^{B}$ Consultant dermatologist, St John's Institute of Dermatology, London, UK; ' 'professor of medical dermatology, St John's Institute of Dermatology, London, UK
An estimated 60 million people have psoriasis worldwide, with country-specific prevalence varying between $0.05 \%$ of general population in Taiwan and $1.88 \%$ in Australia. ${ }^{3.4}$ It is more common in high income areas and those with older populations. ${ }^{3}$ In the UK, it affects $1.52 \%$ of the general population.

\section{Aetiology}

The pathogenesis of psoriasis is multifactorial, with genetics being a primary contributor especially in those with early-onset $(<40$ years) plaque psoriasis. This was demonstrated by twin, familybased and large-scale population-level studies, with heritability estimated to be $60-90 \% .{ }^{5}$ More than 60 susceptibility loci have now been identified using genome-wide association studies. ${ }^{5}$ Many of the candidate causal genes are involved in antigen presentation (HLA-C and ERAP1), NF-kappa B signalling (TNIP1), type 1 interferon pathway (RNF113 and IFIH1), interleukin (IL)23/Th17 axis (IL23R, IL12B and TYK2) and skin barrier function (LCE3). ${ }^{5}$ This suggests a complex interplay between $T$ cells,

\section{Key points}

Psoriasis is a chronic immune-mediated inflammatory skin disease with multiple phenotypically distinct subtypes eg plaque, flexural, guttate, pustular or erythrodermic.

Psoriasis has a major genetic component, with heritability estimated to be $60-90 \%$.

High-impact and difficult-to-treat psoriasis sites include scalp, face, nails, genitalia, palms and soles.

Recognition and management of comorbidities (such as psoriatic arthritis, psychological, cardiovascular and hepatic diseases) is an essential part of holistic care for individuals with psoriasis.

Treatments for psoriasis include topical therapies (vitamin D analogue and corticosteroids), phototherapy (narrowband ultraviolet $B$ radiation (NB-UVB) and psoralen and ultraviolet A radiation (PUVA)), conventional systemic agents (methotrexate, ciclosporin and acitretin), targeted biologics (tumour necrosis factor (TNF), interleukin (IL)-17 and IL-23 inhibitors) and oral small molecule inhibitors (dimethyl fumarate and apremilast).

KEYWORDS: psoriasis, plaque, pustular, multimorbidity, biologic

DOI: 10.7861/clinmed.2021-0257 


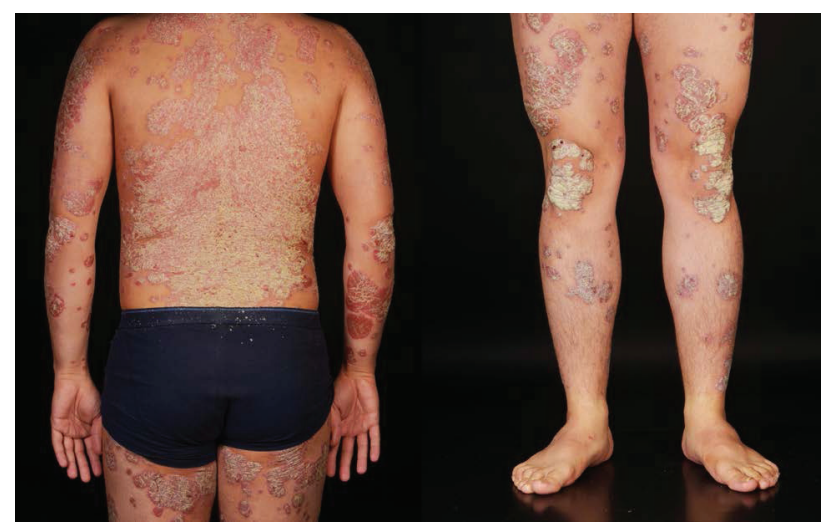

Fig 1. Chronic plaque psoriasis. Widespread, symmetrically distributed and well-demarcated erythematous, scaling plaques. Extensor surfaces such as elbows and knees are typically affected.

dendritic cells and keratinocytes as the likely underlying the pathophysiology of psoriasis, with the IL-23/Th17 axis being the central driver of immune activation, chronic inflammation and keratinocyte proliferation. ${ }^{6}$ Environmental triggers have been known to exacerbate psoriasis such as obesity, stress, betablockers, smoking and lithium.?

Although there is a relative paucity of data, pustular psoriasis appears to be genetically distinct, with different susceptibility genes implicated (IL36RN, AP1S3 in those of European descent and (ARD14). ${ }^{5,8}$

\section{Clinical presentations}

Psoriasis manifests in several ways: plaque, flexural, guttate, pustular or erythrodermic psoriasis. The most common form is plaque psoriasis, which presents as well-demarcated salmon pink plaques with silvery-white scale, typically in a symmetrical distribution and affecting the extensor surfaces (especially elbows and knees), trunk and scalp (Fig 1). Bleeding points may be noted where scales have been removed (Auspitz sign). Flexural psoriasis presents without much scaling and may affect the axillae, sub-mammary and genital areas. Guttate psoriasis causes an acute symmetrical eruption of drop-like papules/ plaques mainly involving the trunk and limbs, that is classically but not always preceded by streptococcal infection. Patients with guttate psoriasis may later develop plaque psoriasis. In rare cases of severe uncontrolled disease, psoriasis causes a widespread erythematous rash (erythroderma) that is life-threatening due to potential complications including hypothermia, risk of infection, acute kidney injury and high-output cardiac failure. Koebner phenomenon describes the appearance of psoriasis at skin areas affected by trauma.

Nails may be affected in up to $50 \%$ of patients and may manifest as nail pitting (indentation in the nails), onycholysis (separation of nail plate from nail bed), oil spots (discoloration of the nailbed), dystrophy and subungual hyperkeratosis.

\section{Multimorbidity and psoriasis}

Defined as the presence of two or more chronic conditions, multimorbidity is common in individuals with psoriasis. Psoriatic arthritis (PsA) affects up to $30 \%$ patients with psoriasis, being more common in those with nail dystrophy and scalp/intergluteal/ perianal psoriasis. ${ }^{9}$ PsA is a heterogeneous disease that may present as a seronegative asymmetric oligoarthropathy, enthesitis or dactylitis. In most patients, psoriasis precedes joint disease by up to 10 years. Thus, general practitioners and dermatologists looking after patients with psoriasis are well-placed to make an early diagnosis of PsA. A validated five-item questionnaire, the Psoriasis Epidemiology Screening Tool (PEST), is recommended to screen for PsA. ${ }^{10}$

Individuals with psoriasis are more likely to suffer from obesity, cardiovascular disease, non-alcoholic fatty liver disease, diabetes and metabolic syndrome than the general population, with rates being especially elevated in those with more severe psoriasis. ${ }^{11}$ This may be related to shared genetic traits, pathogenic inflammatory pathways and common risk factors. ${ }^{12,13}$ The consequence is an elevated mortality rate in patients with severe psoriasis, primarily due to cardiovascular causes. ${ }^{14}$ This is potentially modifiable, with aggressive psoriasis treatment shown to improve cardiovascular outcomes. ${ }^{15}$

In addition, the rates of mental health disorders (such as anxiety and depression) are also elevated compared with the general population, highlighting the psychosocial impact of psoriasis. ${ }^{16}$

\section{Assessment of patients with psoriasis}

Psoriasis is assessed by the extent of skin involvement (body surface area (BSA)) and the severity of erythema, induration and scaling. In secondary care, validated scores such as Psoriasis Area Severity Index (PASI) and Physician Global Assessment Scale are routinely used along with patient reported outcome measures such as Dermatology Life Quality Index (DLQI).1718 Attention to its psychological impact is essential as this may contribute to disengagement and non-adherence to therapy. Every patient encounter is also an opportunity to screen for multimorbidities. In addition to improving overall health, recognition of multimorbidities may influence psoriasis treatment choice. For instance, chronic liver disease may contraindicate methotrexate use. ${ }^{19} \mathrm{~A}$ multidisciplinary approach is, therefore, crucial and often involves rheumatologists, hepatologists and clinical psychologists.

\section{Treatment for psoriasis}

Therapeutic options for psoriasis include topical therapy, phototherapy or systemic treatment, and are summarised in UK National Institute for Health and Care Excellence (NICE) guidelines. ${ }^{10}$ Treatment targets include at least $75 \%$ or $90 \%$ improvement in PASI (PASI75 or PASI90), translating to absolute PASI scores of $\leq 4$ or $\leq 2$, respectively. ${ }^{20}$ Topical therapies such as vitamin D analogues (calcipotriol) or corticosteroids are first line. The efficacy of topical treatment can be increased with occlusion or combination therapy (eg calcipotriol/betamethasone). Previously popular dithranol and tar preparations are less frequently used as they stain and irritate the skin. Psoriasis at difficult-to-treat sites (scalp, face, nails, genitalia, palms and soles) warrants special attention due to its profound impact on function and relatively poor response to treatment (Fig 2). Steroids use for face or genitalia should be of low potency and limited to short-term use due to risk of skin atrophy and telangiectasia.

Second-line therapy includes phototherapy (narrowband ultraviolet $B$ radiation (NB-UVB) and psoralen with ultraviolet $A$ radiation (PUVA)) and conventional systemic agents (methotrexate, 


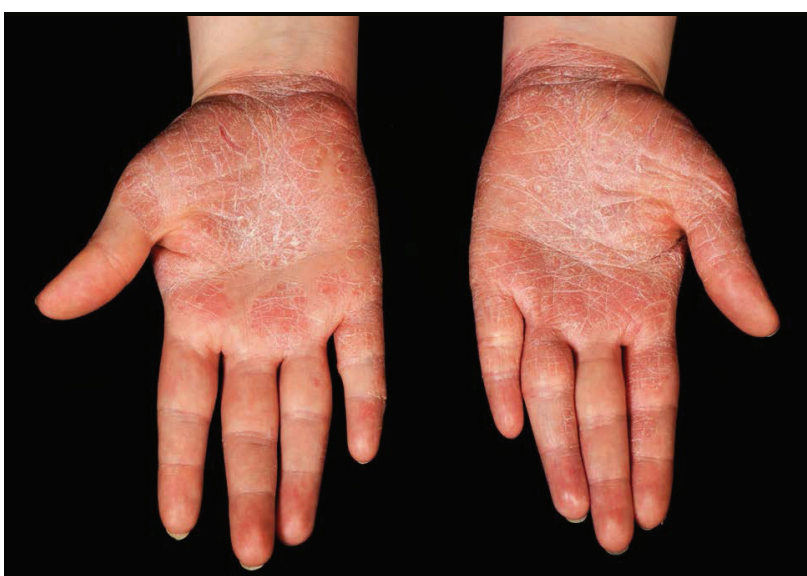

Fig 2. Psoriasis affecting the palms. Psoriasis on the palms, soles, scalp, face, nails and genitalia is difficult to treat and may have a profound impact on activities of daily living.

ciclosporin and acitretin). NB-UVB has largely superseded PUVA due to risks of skin cancer with cumulative doses of PUVA. ${ }^{21}$

Methotrexate works by inhibiting lymphocytes via multiple mechanisms including dihydrofolate reductase inhibition, aminoimidazole carboxamide ribotide transformylase (AICARTase) blockade and adenosine accumulation. Its most serious adverse effect is bone marrow suppression. Other potential complications of treatment include nausea, pneumonitis, hepatitis, liver fibrosis and teratogenicity. Methotrexate is usually taken orally every week. Subcutaneous formulation causes less gastrointestinal side effects and is more efficacious due to higher bioavailability. ${ }^{22}$ Ciclosporin is a calcineurin inhibitor and has a rapid onset of action, but may cause hypertension and irreversible renal toxicity. Acitretin is an oral retinoid that promotes keratinocyte differentiation. Its possible side effects include dry skin, hair loss, hyperlipidaemia and hepatotoxicity. Methotrexate and acitretin are contraindicated in pregnancy. ${ }^{23}$ For disease refractory to methotrexate and/or ciclosporin or where second-line therapies are not suitable, biologic therapies or oral small molecule inhibitors may be considered.

Biologics are monoclonal antibodies or soluble receptors that target proinflammatory cytokines. They have had a dramatic impact on outcomes in moderate-severe disease. Multiple biological therapies are approved for use in moderate-severe psoriasis such as TNF (adalimumab, etanercept, infliximab and certolizumab), IL-12/23p40 (ustekinumab), IL-23p19 (rizankizumab, guselkumab and tildrakizumab), IL-17 (ixekizumab and secukinumab), and IL-17 receptor (brodalumab) inhibitors. There is no single 'best' agent and the choice of biologic needs to be tailored to the needs of each patient. ${ }^{24,25}$ Currently, this is primarily influenced by clinical information eg psoriasis factors (disease phenotype and presence of PsA and outcomes of previous biologic treatment), comorbidities (demyelinating disease and inflammatory bowel disease), drugspecific factors (dosing frequency) and lifestyle considerations (conception plans). ${ }^{24}$ Genomic information has the potential to guide effective deployment of therapies in the future, and this is a field of active research. ${ }^{26}$

Although highly effective, biologics require regular subcutaneous or intravenous administration. Oral small molecule inhibitors including apremilast (phosphodiesterase 4 inhibitor) and dimethyl fumarate are licensed for use in moderate-severe psoriasis, and trials are ongoing for small molecules blocking tyrosine kinase 2 in the Janus kinase (JAK) - signal transducer and activator of transcription proteins (STAT) pathway.

\section{Pustular psoriasis}

Pustular psoriasis is a distinct phenotype characterised by sterile pustules, which can either be acute generalised (generalised pustular psoriasis (GPP)) or limited to digits (acrodermatitis continua of Hallopeau (ACH)) or palms and soles (palmoplantar pustulosis (PPP)). GPP can present acutely with a widespread eruption of superficial pustules and erythematous skin. Patients may be unwell with fever, and blood tests typically show neutrophilia and elevated inflammatory markers. ${ }^{27}$ While GPP can be life-threatening, localised pustulosis can also severely impact on day-to-day activities.

Despite a burgeoning arsenal of treatment for plaque psoriasis, effective treatment for pustular psoriasis remains an area of high unmet need. PPP and ACH are notoriously recalcitrant to the treatments used in plaque psoriasis. Potent topical steroids with occlusion are first line. PUVA may be considered in palmoplantar pustulosis but systemic treatments are frequently required..$^{10}$ For acute severe GPP, ciclosporin or infliximab may be required for its rapid onset of action. Advances in our understanding of the pathogenic role of IL36RN mutations in GPP have also led to the development of IL-36 receptor inhibitors, with trials ongoing. ${ }^{28,29}$

\section{Conclusion}

In summary, psoriasis is a common inflammatory skin condition that is predominantly genetically determined and is associated with significant medical and psychosocial comorbidities. Advances in the understanding of its pathophysiology have led to an increasing number of therapeutic options that could dramatically improve the lives of individuals with psoriasis.

\section{Funding}

Satveer K Mahil and Jonathan N Barker are partly supported by the National Institute for Health Research Biomedical Research Centre at Guy's and St Thomas's NHS Foundation Trust and King's College London.

Satveer K Mahil is funded by a Medical Research Council Clinical Academic Research Partnership award (MR/T02383X/1).

\section{References}

1 World Health Organization. Global report on psoriasis. World Health Organization, 2016.

2 Hay SI, Abajobir AA, Abate KH et al. Global, regional, and national disability-adjusted life-years (DALYs) for 333 diseases and injuries and healthy life expectancy (HALE) for 195 countries and territories, 1990-2016: A systematic analysis for the Global Burden of Disease Study 2016. Lancet 2017:390:1260-344.

3 Parisi R, Iskandar IYK, Kontopantelis E et al. National, regional, and worldwide epidemiology of psoriasis: Systematic analysis and modelling study. BMJ 2020;369:m1590.

4 Global Psoriasis Atlas. Statistics. GPA. http://global-psoriasis-atlas. apos2.swiss 4ward.com/statistics/statistics\#KeyMessages [Accessed 15 March 2021]. 
5 Dand N, Mahil SK, Capon F et al. Psoriasis and genetics. Acta Derm Venereol 2020;100:adv00030.

6 Schön MP, Erpenbeck L. The interleukin-23/interleukin-17 axis links adaptive and innate immunity in psoriasis. Front Immunol 2018:9:1323

7 Budu-Aggrey A, Brumpton B, Tyrrell ] et al. Evidence of a causal relationship between body mass index and psoriasis: A mendelian randomization study. PLoS Med 2019;16:e1002739.

8 Onoufriadis A, Simpson MA, Pink AE et al. Mutations in IL36RN/ IL1F5 are associated with the severe episodic inflammatory skin disease known as generalized pustular psoriasis. Am J Hum Genet 2011;89:432-7.

9 Wilson FC, Icen M, Crowson CS et al. Incidence and clinical predictors of psoriatic arthritis in patients with psoriasis: A populationbased study. Arthritis Care Res. 2009;61:233-9.

10 National Institute for Health and Care Excellence. Psoriasis: assessment and management: Clinical guidline [CG153]. NICE, 2017.

11 Takeshita J, Grewal S, Langan SM et al. Psoriasis and comorbid diseases: Epidemiology. J Am Acad Dermatol 2017;76:377-90.

12 Lonberg AS, Skov L, Skytthe A et al. Association of psoriasis with the risk for type 2 diabetes mellitus and obesity. JAMA Dermatol 2016;152:761-7.

13 Koch M, Baurecht $\mathrm{H}$, Ried JS et al. Psoriasis and cardiometabolic traits: Modest association but distinct genetic architectures. J Invest Dermatol 2015;135:1283-93.

14 Dhana A, Yen H, Yen H, Cho E. All-cause and cause-specific mortality in psoriasis: A systematic review and meta-analysis. J Am Acad Dermatol 2019;80:1332-43.

15 Yang Z sheng, Lin N ning, Li L, Li Y. The Effect of TNF Inhibitors on Cardiovascular Events in Psoriasis and Psoriatic Arthritis: an Updated Meta-Analysis. Clin Rev Allergy Immunol 2016;51:240-7.

16 Kurd SK, Troxel AB, Crits-Christoph P, Gelfand JM. The risk of depression, anxiety, and suicidality in patients with psoriasis: A population-based cohort study. Arch Dermatol 2010;146:891-5.

17 Finlay AY, Khan GK. Dermatology Life Quality Index (DLQI) —a simple practical measure for routine clinical use. Clin Exp Dermatol 1994:19:210-6.

18 Feldman SR, Krueger GG. Psoriasis assessment tools in clinical trials. Ann Rheum Dis 2005;64(Suppl 2):ii65-8.

19 Puig L. Obesity and psoriasis: Body weight and body mass index influence the response to biological treatment. J Eur Acad Dermatol Venereol 2011;25:1007-11.
20 Mahil SK, Wilson N, Dand N et al. Psoriasis treat to target: defining outcomes in psoriasis using data from a real-world, populationbased cohort study (the British Association of Dermatologists Biologics and Immunomodulators Register, BADBIR). Br J Dermatol 2020;182:1158-66

21 Ling TC, Clayton TH, Crawley ] et al. British Association of Dermatologists and British Photodermatology Group guidelines for the safe and effective use of psoralen-ultraviolet A therapy 2015. Br J Dermatol 2016;174:24-55.

22 Warren RB, Weatherhead SC, Smith CH et al. British Association of Dermatologists' guidelines for the safe and effective prescribing of methotrexate for skin disease 2016. Br J Dermatol 2016;175:23-44.

23 Nast A, Smith C, Spuls PI et al. EuroGuiDerm Guideline on the systemic treatment of Psoriasis vulgaris - Part 2: specific clinical and comorbid situations. J Eur Acad Dermatology Venereol 2021;35:281-317.

24 Smith CH, Yiu ZZN, Bale T et al. British Association of Dermatologists guidelines for biologic therapy for psoriasis 2020: a rapid update. $\mathrm{Br}$ J Dermatol 2020;183:628-37.

25 Mahil SK, Ezejimofor MC, Exton LS et al. Comparing the efficacy and tolerability of biologic therapies in psoriasis: an updated network meta-analysis. Br ] Dermatol 2020;183:638-49.

26 Dand N, Duckworth M, Baudry D et al. HLA-C*06:02 genotype is a predictive biomarker of biologic treatment response in psoriasis. J Allergy Clin Immunol 2019;143:2120-30.

27 Benjegerdes K, Hyde K, Kivelevitch D, Mansouri B. Pustular psoriasis: pathophysiology and current treatment perspectives. Psoriasis (Auckl) 2016;6:131-44.

28 Bachelez H, Choon S-E, Marrakchi S et al. Inhibition of the interleukin-36 pathway for the treatment of generalized pustular psoriasis. N Engl J Med 2019;380:981-3.

29 Mahil SK, Catapano M, Di Meglio P et al. An analysis of IL-36 signature genes and individuals with IL1RL2 knockout mutations validates IL-36 as a psoriasis therapeutic target. Sci Transl Med 2017;9:eaan2514.

Address for correspondence: Prof Jonathan N Barker, St John's Institute of Dermatology, King's College London, 9th Floor Tower Wing, Guy's Hospital, London SE1 9RT, UK. Email: jonathan.barker@kcl.ac.uk 